EPJ Web of Conferences 70, 00020 (2014)

DOI: $10.1051 /$ epjconf/ 20147000020

(C) Owned by the authors, published by EDP Sciences, 2014

\title{
Highlights from the ATLAS Experiment
}

\author{
Sally Seidel ${ }^{1}$, on behalf of the ATLAS Collaboration \\ ${ }^{1}$ Department of Physics and Astronomy, University of New Mexico, MSC 07 4220, Albuquerque, NM 87131, USA
}

Abstract. New results by the ATLAS experiment at the LHC in top quark studies, investigations of Standard Model processes, Higgs searches, and searches for phenomena beyond the Standard Model are presented.

\section{Introduction}

ATLAS [1], one of the four experiments at the CERN Large Hadron Collider, has produced a wealth of new results in 2012 on topics in top quark studies, Standard Model processes, Higgs searches, and searches for phenomena beyond the Standard Model (BSM). A sample of measurements based on data collected in 2010-2011 is described here.

\section{Standard Model Investigations}

A measurement of inclusive two-particle angular correlations has been made [2]. The study uses charged particles of transverse momentum $\mathrm{p}_{\mathrm{T}}>100 \mathrm{MeV}$ and pseudorapidity magnitude $|\eta|<2.5$ collected with minimum bias triggers. Measurements at center of mass energies $\sqrt{s}_{\mathrm{s}}=900 \mathrm{GeV}$ and $7 \mathrm{TeV}$ are compared with predictions by the PYTHIA 8 and HERWIG++ generators and 3 tunes of the PYTHIA 6 generator. None of the models satisfactorily describe the data, a result that impacts the phenomenology of soft particle production including models for diffraction and hadronization. Addressing the situation may go beyond retuning existing models. Figure 1 shows an example of the data to theory comparison for one choice of multiplicity and $\eta$ interval.

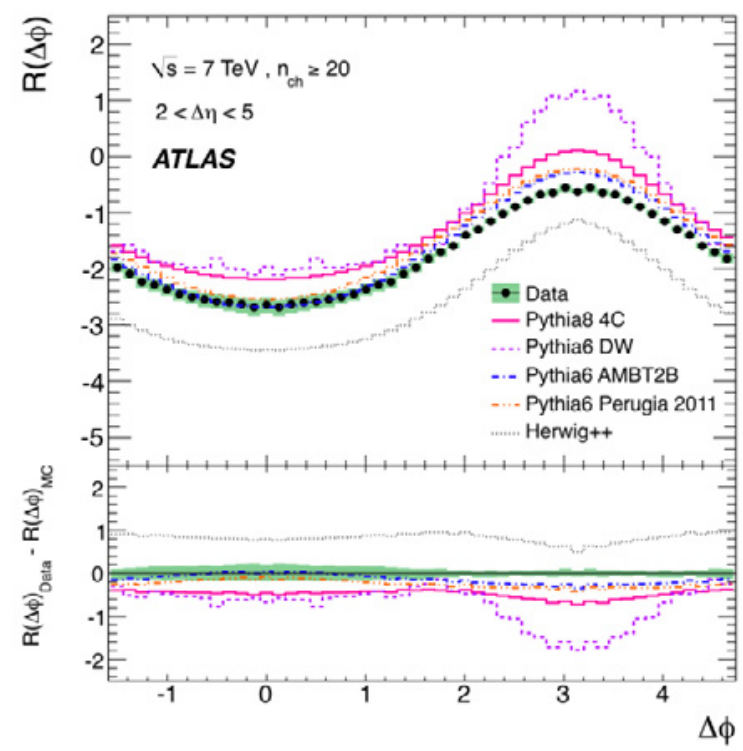

Fig. 1. Corrected $\mathrm{R}(\Delta \phi)$ two-particle correlation functions obtained by integrating the foreground and background distributions over $\Delta \eta$ between 2 and 5 for data and the indicated Monte Carlo generators for charge multiplicity $\geq 20$ [2]. 
The measurement of the azimuthal ordering of charged hadrons [3] tests models of QCD at low energy scales, typically with non-perturbative contributions. Predictions based on the Lund model roughly reproduce the inclusive power spectra observed in the $\sqrt{s}_{\mathrm{s}}=900 \mathrm{GeV}$ and $7 \mathrm{TeV}$ data. The models systematically overestimate correlations, especially in phase space regions dominated by diffractive events. Inclusion of azimuthally ordered fragmentation (helically ordered gluon chains) may improve the models of fragmentation and soft production. Figure 2 shows an example of the comparison between data and theory for the $S_{\eta}$ power spectrum with enhanced soft component.

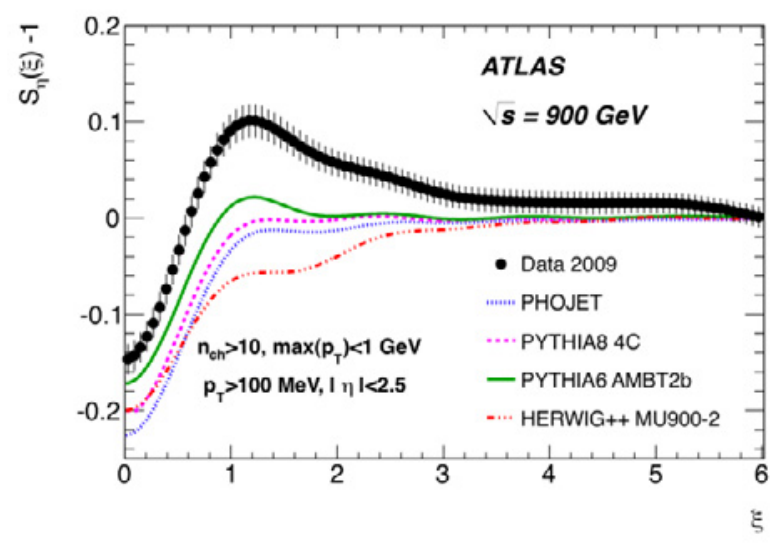

Fig. 2. Corrected data compared to particle level predictions from various Monte Carlo models using conventional hadronization algorithms, for the $S_{\eta}$ power spectrum and event selection with enhanced soft component $\left(\mathrm{p}_{\mathrm{T}}>100 \mathrm{MeV}, \max \left(\mathrm{p}_{\mathrm{T}}\right)<1 \mathrm{GeV}\right)$ [3]. The error bars correspond to the combined statistical and systematic uncertainties.

The measurement of the differential cross sections for the processes $W \rightarrow \ell v$ and $Z \rightarrow \ell \ell$ [4] points to the existence of a flavor-symmetric light quark sea at low $x$. ATLAS data combined with HERA ep results place new constraints on the strange quark distribution at scale $Q^{2} \sim M_{Z}^{2}$, and at low $Q^{2}$ by perturbative QCD evolution. This study provides new sensitivity to the $s$ quark density at $x \sim 0.01$. The value of the ratio $r_{x}=(s(x)+\bar{s}(x)) / 2 \bar{d}(x)$ is $1.00_{-i \leq b}^{+\infty}$ at $x_{B j}=0.023$ and $Q^{2}=1.9 \mathrm{GeV}^{2}$. Figure 3 shows the distribution of light sea quarks as a function of $x$ for two choices of strangeness fraction.

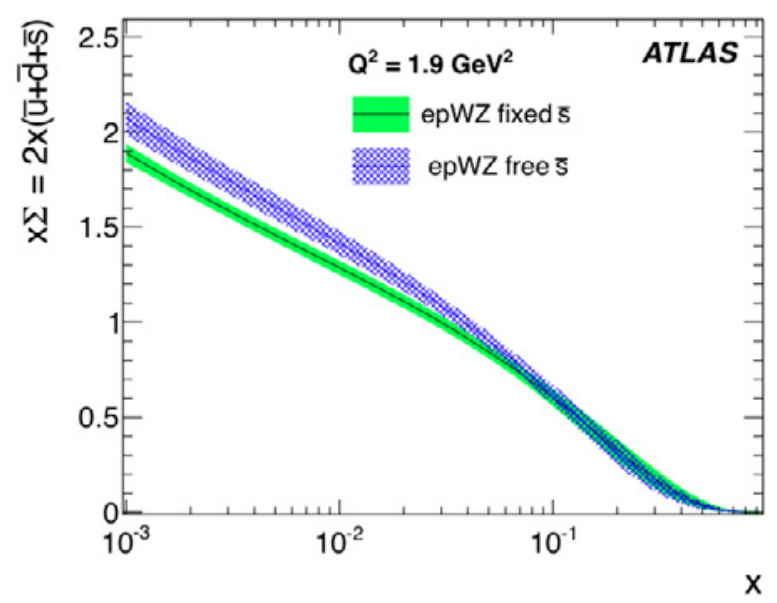

Fig. 3. Distribution of the light sea quarks in the NNLO analysis of HERA and ATLAS data [4] with a fixed fraction of strangeness (lower, green curve) and with a fitted fraction of about unity (upper, blue curve). The bands represent experimental uncertainties.

The production cross section of an isolated photon with jets has been measured [5]. Prompt photon production tests perturbative QCD at large hard scattering scales and over a wide range of parton momentum fraction $x$. Photon-jet angular correlations constrain the photon fragmentation functions. Since the dominant $\gamma+$ jet production mechanism in pp collisions at the LHC is through the $q g \rightarrow q \gamma$ process, this measurement at high rapidities and low transverse momenta can also be used to constrain the gluon density function inside the proton. Additionally this measurement is important as a background to the process $H \rightarrow \gamma \gamma$. The JETPHOX generator is found to agree well with the ATLAS data except in the region $\mathrm{E}_{\gamma}<45 \mathrm{GeV}$. Figure 4 shows the photon + jet production cross section measurement and theoretical prediction in the interval $2.8 \leq\left|\mathrm{y}^{\mathrm{jet}}\right|<4.4$. 


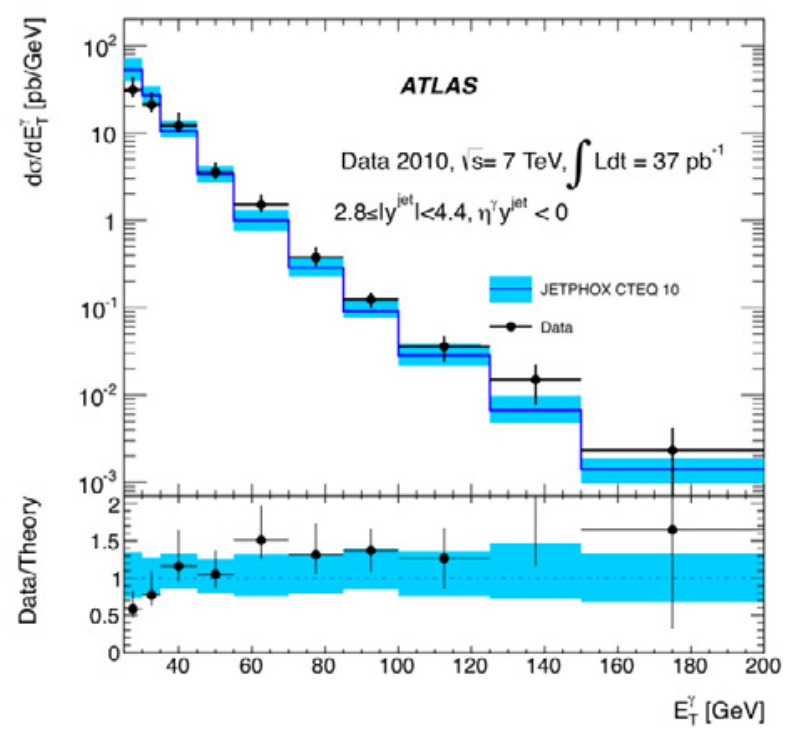

Fig. 4. Top graph: Experimental (black dots) and theoretical (blue line) photon + jet production cross section, for production in opposite pseudorapidity hemispheres, with $2.8 \leq\left|y^{\mathrm{jet}}\right|<4.4$ [5]. The black error bars represent the total experimental uncertainty. The blue bands show the total uncertainty on the theoretical prediction obtained with JETPHOX. Bottom graph: ratio between the measured and predicted cross sections. The blue bands show the theoretical uncertainties while the error bars show the experimental uncertainties on the ratio.

\section{Top as a Probe Beyond the Standard Model}

A measurement of the charge asymmetry $A_{C}$ in top quark pair production has been made [6] using the definition

$A_{C}=\frac{N(\Delta|y|>0)-N(\Delta|y|<0)}{N(\Delta|y|>0)+N(\Delta|y|<0)}$,

where $\Delta|y| \equiv\left|y_{t}\right|-\left|y_{\bar{t}}\right|$ is the difference between the top and anti-top rapidities. The data set includes events with a single lepton, missing $\mathrm{p}_{\mathrm{T}}$, a $b$-jet, and at least 3 additional jets. The measured value $A_{C}=-0.018 \pm 0.028 \pm 0.002$ is consistent with the prediction by the MC@NLO package of 0.006 \pm 0.002 . This measurement and an equivalent one by CMS [7] are now in tension with Tevatron forward-backward asymmetry measurements [8] as well as new models that assume the existence of a $W^{\prime}$ or $Z^{\prime}$. Figure 5 shows these experimental results as well as predictions by the Standard Model and new physics models.

A search has been conducted for flavor-changing neutral current single top production [9]. This process is suppressed in the Standard Model by the GIM mechanism, so observation of it would signal new physics. Top is sought in its semileptonic decay. The ATLAS limits that result for coupling (Figure 6) and branching ratio (Figure 7) are the most stringent to date on the process $q g$ $\rightarrow t$. 


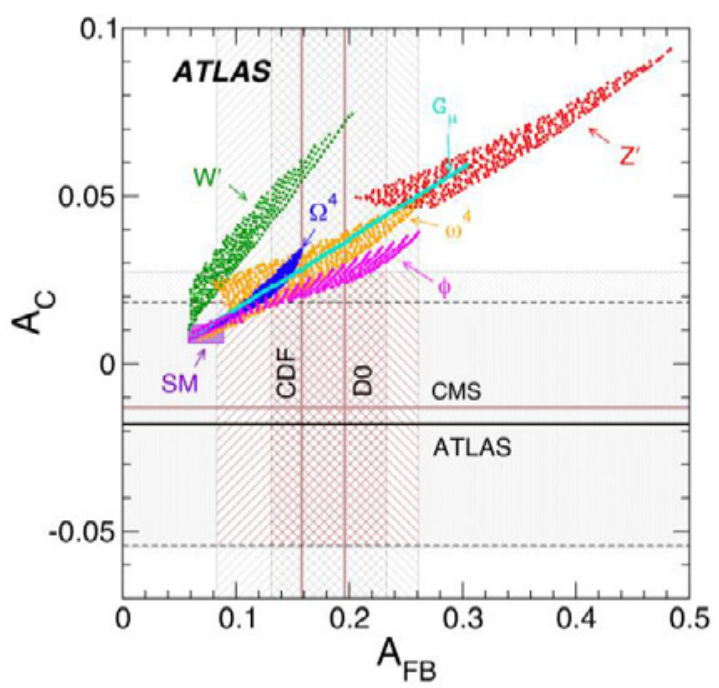

Fig. 5. Measured forward-backward asymmetries from the Tevatron and charge asymmetries from the LHC, compared to predictions from the Standard Model as well as predictions incorporating various potential new physics contributions [6]. The horizontal (vertical) bands and lines correspond to ATLAS and CMS (CDF and D0) measurements. The inclusive values are presented.

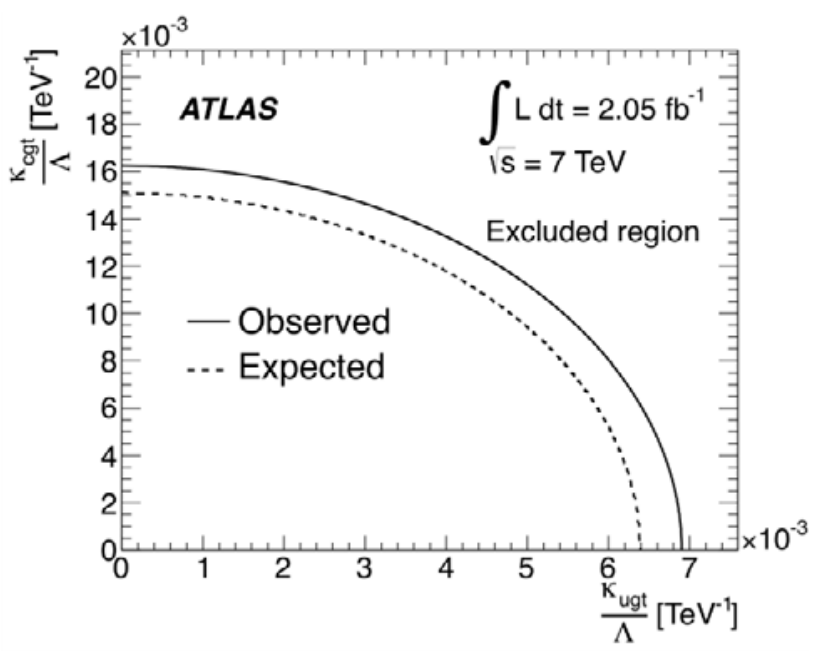

Fig. 6. The upper limits on the coupling constants $\kappa_{\text {ugt }} / \Lambda$ and $\kappa_{\mathrm{cgt}} / \Lambda[9]$.

ATLAS has conducted a search for same-sign top quark production and fourth generation down-type quarks [10]. The data set includes events whose final states have two isolated same-sign leptons, two or more jets, and large missing transverse energy $\mathrm{E}_{\mathrm{T}}{ }^{\text {miss }}$. Limits of $1.7 \mathrm{pb}$ are set at $95 \%$ confidence level (CL) on the cross section for each chirality of the BSM mediators charge-4/3 color triplet $Q_{\mu}{ }^{5}$, color sextet $y_{\mu}{ }^{5}$, charge-neutral color singlet $Z^{\prime}$, and color octet $g^{\prime}$. This is the strongest limit available in the like-sign channel. Figure 8 shows the mass exclusion limits on cross section times branching ratio. 


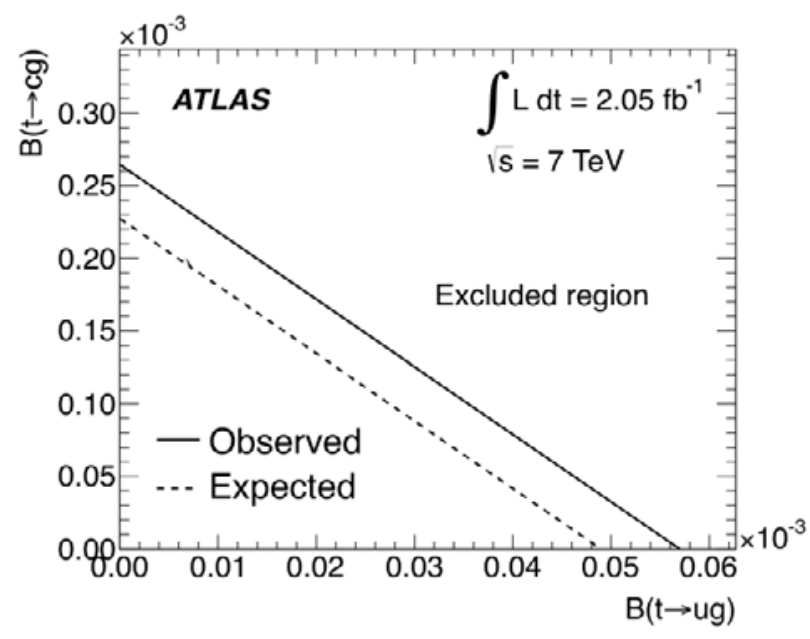

Fig. 7. The upper limits on the branching fractions $t \rightarrow u g$ and $t \rightarrow c g$ [9].

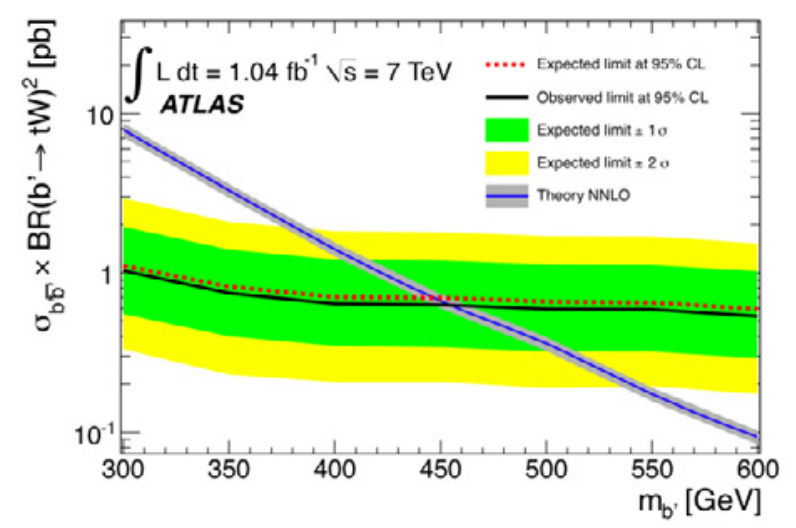

Fig. 8. The $95 \%$ confidence level exclusion limits on cross section times branching ratio for $b^{\prime} \bar{b}^{\prime}$ production with decay $b^{\prime} \rightarrow t W[10]$.

\section{Searches for New Physics}

A search has been conducted for a new quark $b^{\prime}$ decaying through $b^{\prime} \rightarrow Z b$ using events with a $b$-tagged jet and a $Z \rightarrow e^{+} e^{-}$decay [11]. This search excludes $b$ quarks with masses below $400 \mathrm{GeV}$, which is an improvement over the previous limit of $268 \mathrm{GeV}$. Figure 9 shows the cross section limits as a function of $b^{\prime}$ mass.

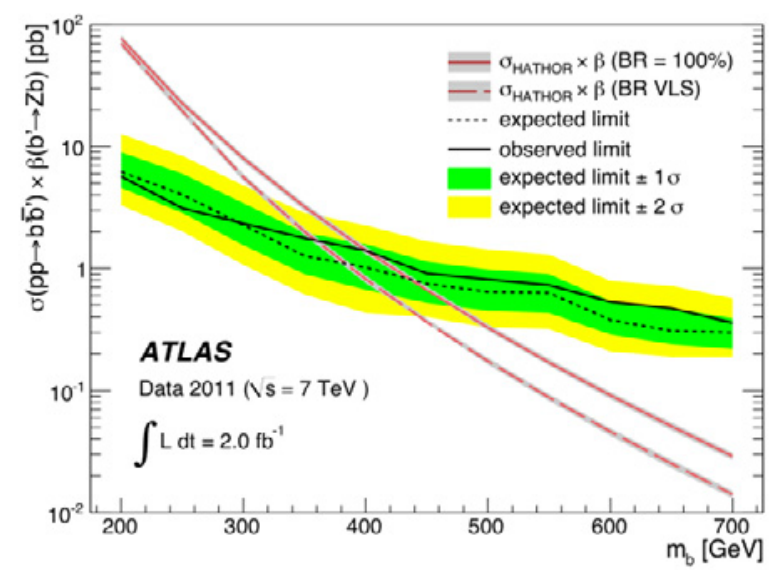

Fig. 9. The observed and expected $95 \% \mathrm{CL}$ cross section limits as a function of $b^{\prime}$ mass for the decay $b^{\prime} \rightarrow Z b$. The signal cross section is shown with uncertainties arising from PDFs and renormalization and factorization scale choice. The prediction is also multiplied by the $\beta$ factors described in Ref. [10]. 
ATLAS has also searched for down-type fourth generation quarks through the process $b^{\prime} \bar{b}^{\prime} \rightarrow W^{-} t W^{+} \bar{t} \rightarrow b \bar{b} W^{+} W W^{+} W^{-} \rightarrow \ell^{*} w b \bar{b} q \bar{q} q \bar{q} q \bar{q}[12]$. The lower limit (Figure 10) on the mass is raised to $480 \mathrm{GeV}$ a significant improvement over the CDF limit [13].

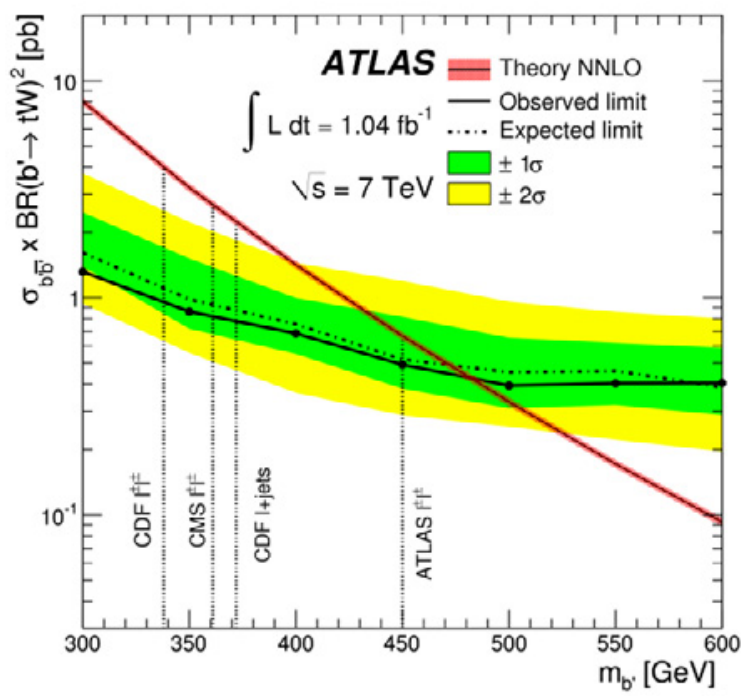

Fig. 10. Observed and expected cross section exclusion upper limits at 95\% CL for a fourth-generation $b^{\prime}$ quark for the decay $b^{\prime} \rightarrow W^{\prime} t[12]$ Systematic uncertainties on the expected limit are shown with shaded bands.

A fourth generation top $t^{\prime}$ enters some theories as a source of CP violation. ATLAS has searched in the lepton + jets channel for pair production of this heavy quark which decays to $W+b$ [14]. Events are required to have one high $\mathrm{p}_{\mathrm{T}}$ isolated $e$ or $\mu$, high missing $\mathrm{p}_{\mathrm{T}}$, and three or more jets. The $95 \%$ CL lower mass limit is now $404 \mathrm{GeV}$ (Figure 11).

Events that include a lepton, missing transverse momentum $\mathrm{p}_{\mathrm{T}}{ }^{\text {miss }}$, and 2 jets are examined for evidence of new right-handed bosons $W_{R}{ }^{\prime}$ that decay through $W_{s}{ }^{\prime} \rightarrow t b \rightarrow \ell v b b$ [15]. The result of the search is the most stringent direct lower limit on the mass, $1.13 \mathrm{TeV}$ at $95 \% \mathrm{CL}$, as shown in Figure 12.

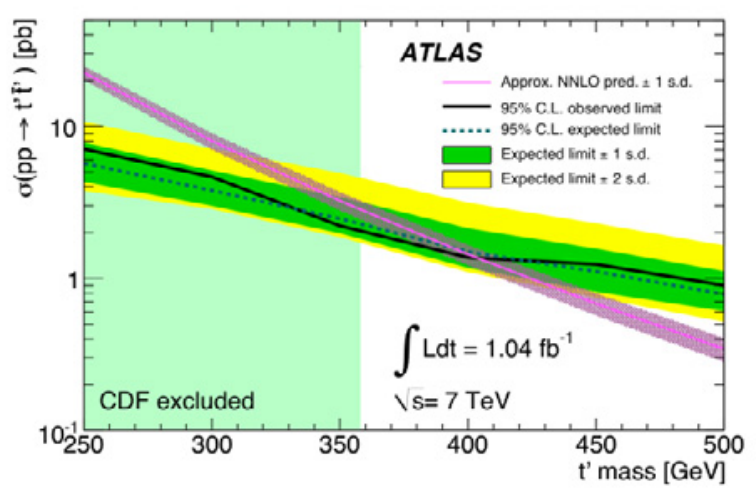

Fig. 11. Observed (solid line) and expected (dashed line) 95\% CL upper limits on the $t^{\prime} \bar{t}$ ' cross section as a function of the $t^{\prime}$ mass [14]. The surrounding shaded bands correspond to 1 and 2 standard deviations around the expected limit. The thin line shows the theoretical prediction including its 1 standard deviation uncertainty band. The shaded area is the mass region previously excluded by the CDF experiment. 


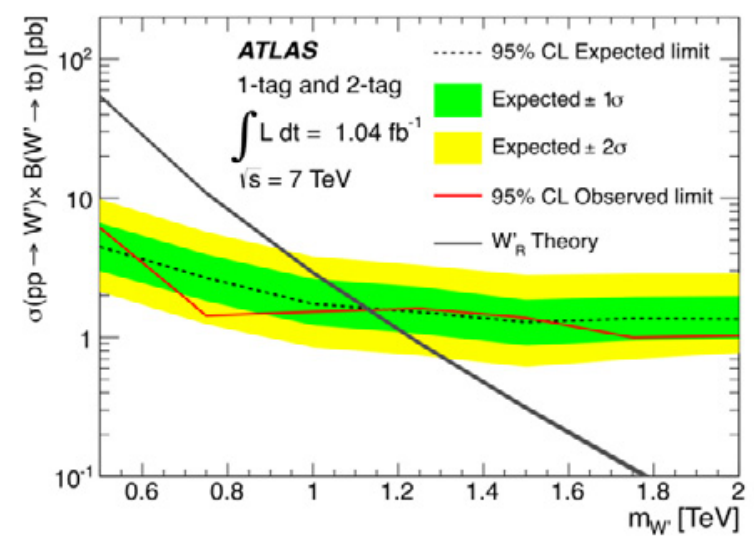

Fig. 12. $95 \% \mathrm{CL}$ limit on the cross section, $\sigma\left(p p \rightarrow W_{R}{ }^{\prime}\right)$, times branching ratio for $W_{R}^{\prime} \rightarrow t b$ as a function of the $W^{\prime}$ boson mass [15]. The theory curve is also shown.

A search has also been made for heavy neutrinos and right-handed new $W$-type bosons [16]. Events with two high-p $\mathrm{p}_{\mathrm{T}}$ leptons and at least one high- $\mathrm{p}_{\mathrm{T}}$ hadronic jet are studied within the scenarios of no mixing and maximal mixing of the $e$ and $\mu$ generations for Majorana and Dirac neutrinos. The limits produced are the most stringent to date from direct searches. Figure 13 shows the limits in the Dirac neutrino case.

Excited leptons have been sought [17] in events with a final state including two identified leptons and a photon that are energetic, isolated, and well separated from each other. Limits at $95 \% \mathrm{CL}$ are set on $\sigma \times B R\left(\ell^{*} \rightarrow \ell \gamma\right)$. For mass $m_{r}>0.9 \mathrm{TeV}$, the limit is $2.3 \mathrm{fb}$ for $e^{*}$ and $4.5 \mathrm{fb}$ for $\mu^{*}$. In the case where the compositeness scale is equal to the mass of the excited lepton, these measurements exclude masses $m_{-}<1.87 \mathrm{TeV}$ and $m_{n+}<1.75 \mathrm{TeV}$. Figures 14 and 15 show the cross section $\times$ branching ratio limits as functions of the masses of the excited electron and muon respectively.

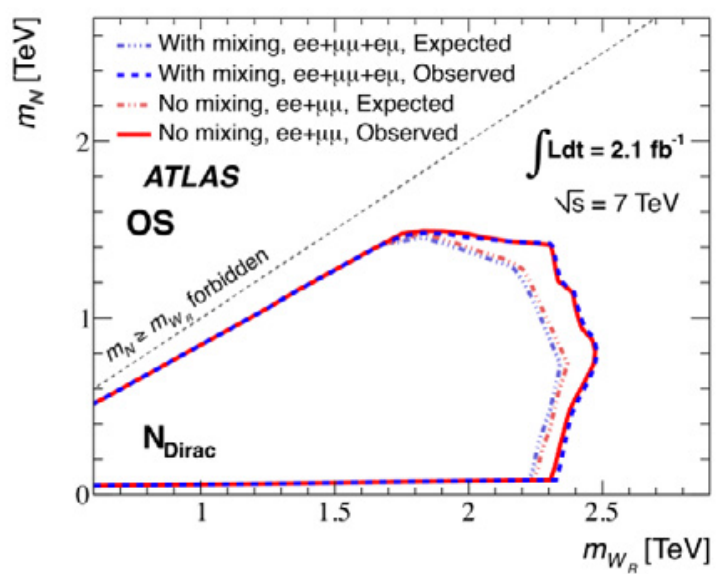

Fig. 13. Observed and expected $95 \%$ CL upper limits on the heavy neutrino and $W_{R}$ masses for the Dirac case, in the no-mixing and maximalmixing scenarios [16]. 


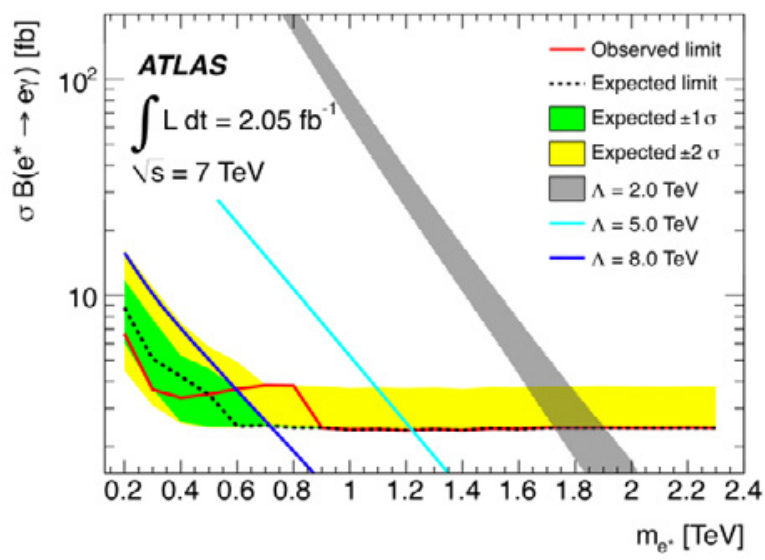

Fig. 14. Cross section $\times$ branching ratio limits at $95 \% \mathrm{CL}$ as a function of $\mathrm{e}^{*}$ mass [17]. Theoretical predictions for excited electrons produced for three different compositeness scales are shown, as well as the theoretical uncertainties from renormalization and factorization scales and PDFs for $\Lambda=2 \mathrm{TeV}$.

A search has also been conducted for second- generation scalar leptoquarks [18], using events with final states of $\mu \mu+$ at least 2 jets or $\mu+\mathrm{E}_{\mathrm{T}}{ }^{\text {miss }}+$ at least 2 jets. The mass range excluded (see Figure 16) depends upon the assumed branching ratio BR for the decay of the leptoquark to $\mu q$. The excluded mass range is $\mathrm{m}_{\mathrm{LQ}}<595(685) \mathrm{GeV}$ at $95 \% \mathrm{CL}$ for $\mathrm{BR}_{\mathrm{LQ} \rightarrow \mu \mathrm{q}}=0.5(1.0)$.

ATLAS has searched for the Randall-Sundrum graviton $\mathrm{G}^{*}$ through decays to $Z Z$ using final states with leptons and jets [19]. The data set is composed of events with 4 charged leptons or 2 charged leptons and 2 jets. This graviton is excluded at $95 \%$ CL in the mass range $325-845 \mathrm{GeV}$ (see Figure 17) for $k / \bar{m}_{m-i}=0.1$, where $k$ is the curvature scale of the warped extra dimension and $\bar{m}=m_{\text {rex }} / \sqrt{8 \pi}$.

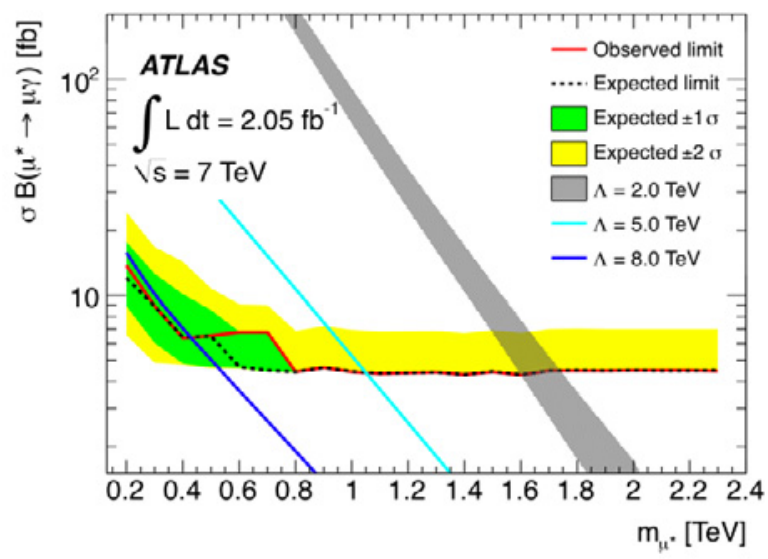

Fig. 15. Cross section $\times$ branching ratio limits at $95 \% \mathrm{CL}$ as a function of $\mu^{*}$ mass [17]. Theoretical predictions for excited muons produced for three different compositeness scales are shown, as well as the theoretical uncertainties from renormalization and factorization scales and PDFs for $\Lambda=2 \mathrm{TeV}$. 


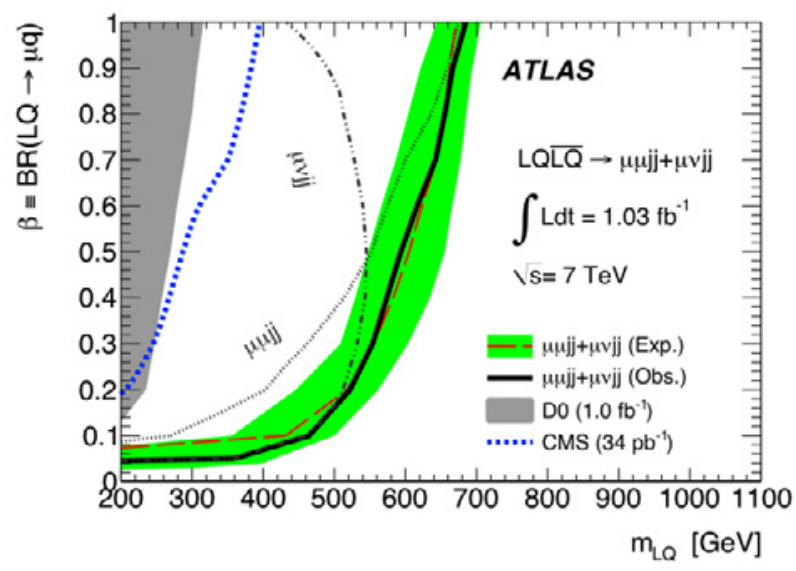

Fig. 16. The $95 \% \mathrm{CL}$ exclusion region resulting from the combination of the $\mu \mu \mathrm{jj}$ and $\mu v \mathrm{jj}$ channels shown in the branching ratio versus leptoquark mass plane [18]. The shaded area at the left indicates the D0 exclusion limit and the thick dotted line indicates the CDF exclusion region. The dotted and dotted-dashed lines indicate the individual limits derived for the $\mu \mu \mathrm{jj}$ and $\mu v \mathrm{jj}$ channels, respectively. The combined observed limit is indicated by the solid black line. The combined expected limit is indicated by the dashed line, together with the solid band containing $68 \%$ of possible outcomes from pseudo-experiments in which the yield is Poisson-fluctuated around the background-only expectation.

The search for contact interactions [20] uses dilepton events with large invariant mass. With a prior flat in $1 / \Lambda^{2}$ (where $\Lambda$ is the energy scale below which fermion constituents are bound), $95 \%$ CL limits are set on the energy scales as follows. For the electron channel, $\Lambda^{-}\left(\Lambda^{+}\right)>10.1(9.4) \mathrm{TeV}$. For the muon channel, $\Lambda^{-}\left(\Lambda^{+}\right)>8.0(7.0) \mathrm{TeV}$. The muon channel limits are the most stringent to date. Figure 18 shows the dimuon invariant mass distribution.

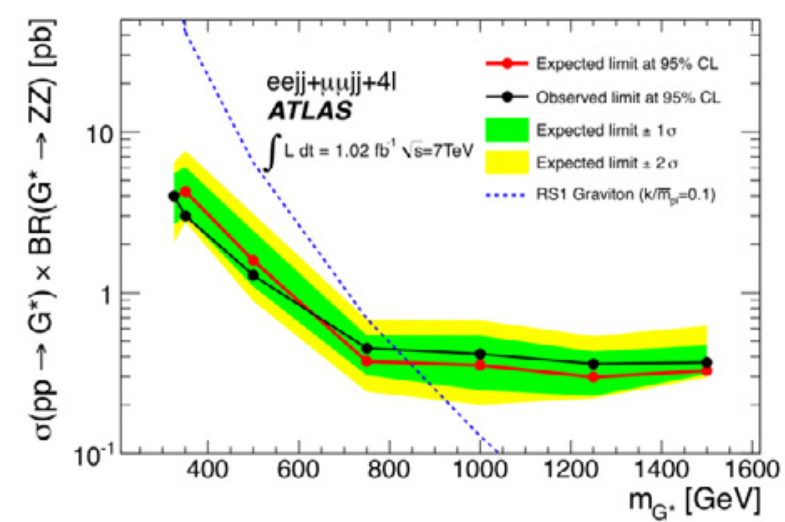

Fig. 17. Observed and expected $95 \%$ CL limits for $\mathrm{G}^{*} \rightarrow \mathrm{ZZ}$ for the combined $11 j j+\mathrm{IIII}$ channels [19]. The leading order theoretical prediction is also shown for $k / \bar{m}_{p l}=0.1$. 


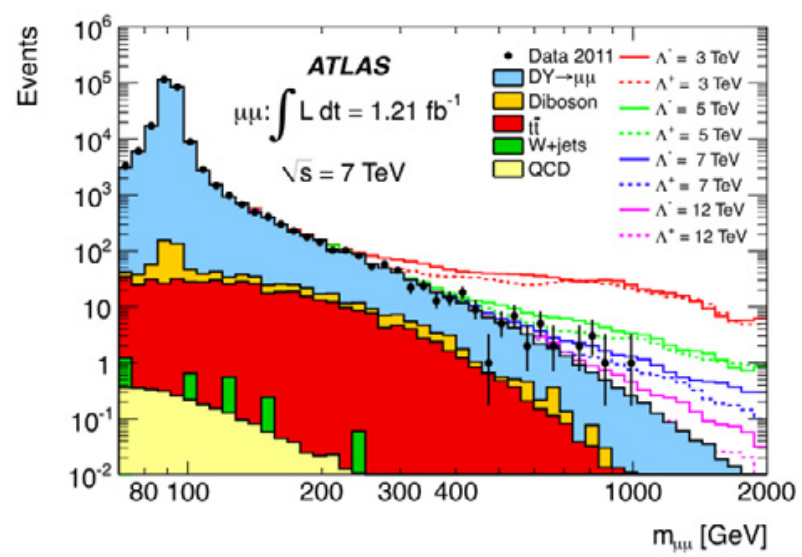

Fig. 18. The dimuon invariant mass distribution for data (points) and Monte Carlo simulation (histograms) [20]. The open histograms correspond to the distributions expected in the presence of contact interactions with different values of $\Lambda$ for both constructive (solid histograms) and destructive (dashed histograms) interference.

Final states with multiple high $\mathrm{p}_{\mathrm{T}}$ particles including charged leptons and jets are used to search for TeV-scale gravity signatures [21]. The semiclassical approximations used in the modelling of black hole production are valid only for partonic center of mass energies well above the Planck scale in $n+4$ dimensions, motivating the use of a minimal threshold $M_{T H}$ to remove contributions where the modelling is not reliable. ATLAS data are compared to predictions by the CHARYBDIS generator to produce exclusion limits in the plane of minimal threshold versus string scale for rotating string balls with 6 extra dimensions (see Figure 19). The data are also compared to predictions by the BLACKMAX generator (Figure 20) to yield exclusion limits in the plane of minimal threshold versus Planck scale in $n+4$ dimensions for rotating black holes with 6 extra dimensions.

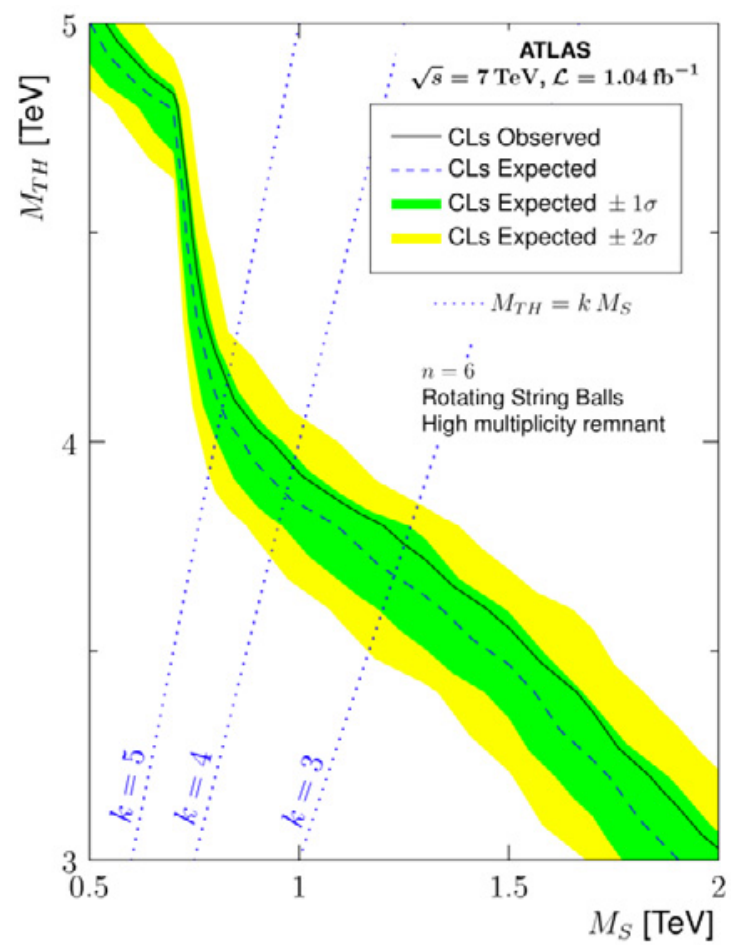

Fig. 19. The exclusion limit in the $\mathrm{M}_{\mathrm{TH}}-\mathrm{M}_{\mathrm{s}}$ plane, with electron and muon channels combined, for rotating string balls with six extra dimensions [21]. The solid (dashed) line shows the observed (expected) $95 \%$ CL limit, with the dark and light bands the expected $1 \sigma$ and $2 \sigma$ variations of the expected limits. (This convention applies to all subsequent figures as well.) The dotted lines indicate constant $\mathrm{k}=\mathrm{M}_{\mathrm{TH}} / \mathrm{Ms}_{\mathrm{s}}$. All samples were produced with the CHARYBDIS generator, using a high multiplicity remnant state.

A search has been conducted for anomalous production of prompt pairs of like-sign muons [22]. Prompt like-sign lepton pairs appear in many BSM theories, and among these, theories of doubly-charged Higgs predict a narrow resonance. The limits on production cross section for the $\mathrm{H}^{++}$that result range from $5.3 \mathrm{fb}$ to $58 \mathrm{fb}$ for dimuon mass in the range $300-15 \mathrm{GeV}$. The upper limit on the mass is $355 \mathrm{GeV}$ or $251 \mathrm{GeV}$ depending upon whether the $\mathrm{H}^{++}$couples to left-handed (Figure 21) or right-handed (Figure 22) fermions, respectively. 


\section{Higgs Searches}

Top events with a final state $\tau$ have been used to search for the charged Higgs $H^{+}$[23]. Assuming that the branching ratio for $H^{+} \rightarrow$ $\tau v$ is $100 \%$, the result, which is consistent with the Standard Model, implies upper limits on the $t \rightarrow H^{+} b$ branching ratio in the range $5 \%$ to $1 \%$ for Higgs mass $m_{H^{+}}$in the range $90-160 \mathrm{GeV}$, as shown in Figure 23. In the MSSM, a value of tan $\beta$ above $12-26$ or between 1 and 2-6 is excluded (Figure 24) for $90 \mathrm{GeV}<m_{H^{+}}<150 \mathrm{GeV}$.

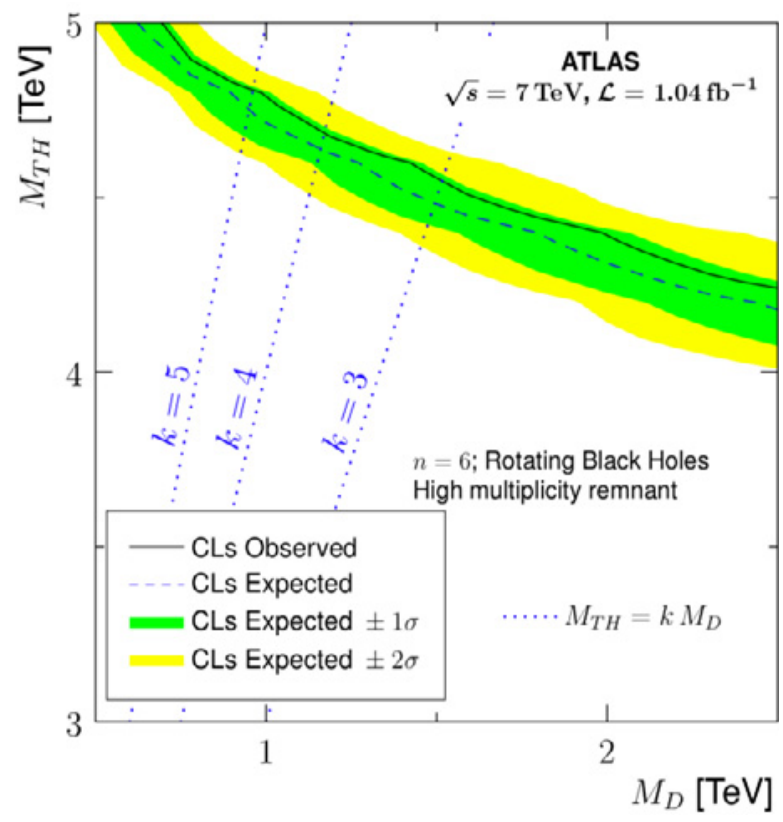

Fig. 20. The exclusion limit in the $\mathrm{M}_{T H}-\mathrm{M}_{\mathrm{D}}$ plane, with electron and muon channels combined, for rotating black hole models with six extra dimensions [21]. The black hole decays result in a high multiplicity remnant state generated with BLACKMAX. The solid (dashed) line shows the observed (expected) $95 \%$ CL limits. The dotted lines indicate constant $\mathrm{k}=\mathrm{M}_{\mathrm{TH}} / \mathrm{MD}_{\mathrm{D}}$.

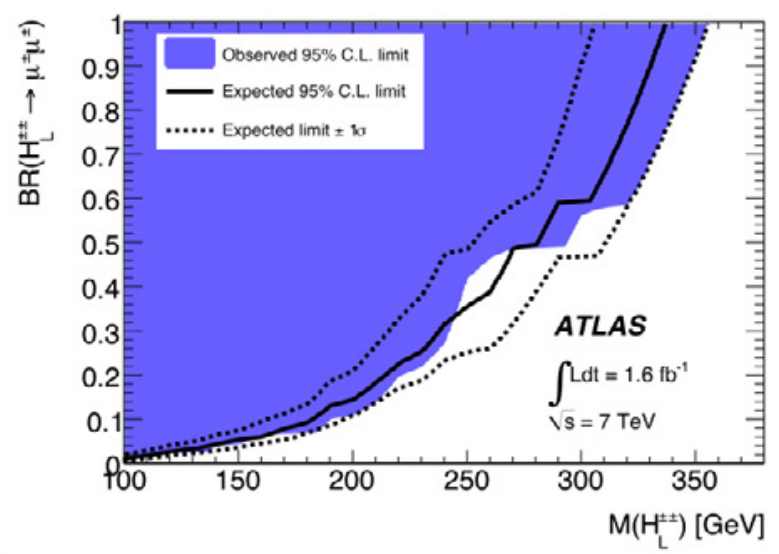

Fig. 21. Exclusion limit at $95 \% \mathrm{CL}$ of the $H^{ \pm \pm}$mass as a function of the branching ratio to muon pairs, $B R\left(H^{ \pm \pm} \rightarrow \mu^{ \pm} \mu^{ \pm}\right)$, for $H_{L}^{ \pm \pm}$bosons. The shaded areas show the observed exclusion region, the solid lines show the expected exclusion region, and the dashed lines show the $\pm 1 \sigma$ variations of the expected exclusion region [22].

ATLAS searches for the Standard Model Higgs in the diphoton decay channel [24]. Each photon has $\mathrm{E}_{\mathrm{T}} \geq 20 \mathrm{GeV}$, and to optimize sensitivity, events are separated into 9 mutually exclusive categories with different mass resolutions and signal-tobackground ratios. Misidentified jets and Drell-Yan electrons that pass the photon selection are backgrounds to this search. An excess of events at mass $126.5 \mathrm{GeV}$ is observed (see Figure 25) with significance $2.8 \sigma$ in $4.9 \mathrm{fb}^{-1}$ of data. Figure 26 shows the observed and expected 95\% CL limits on the Standard Model Higgs boson production normalized to the predicted cross section as a function of $\mathrm{m}_{\mathrm{H}}$. 


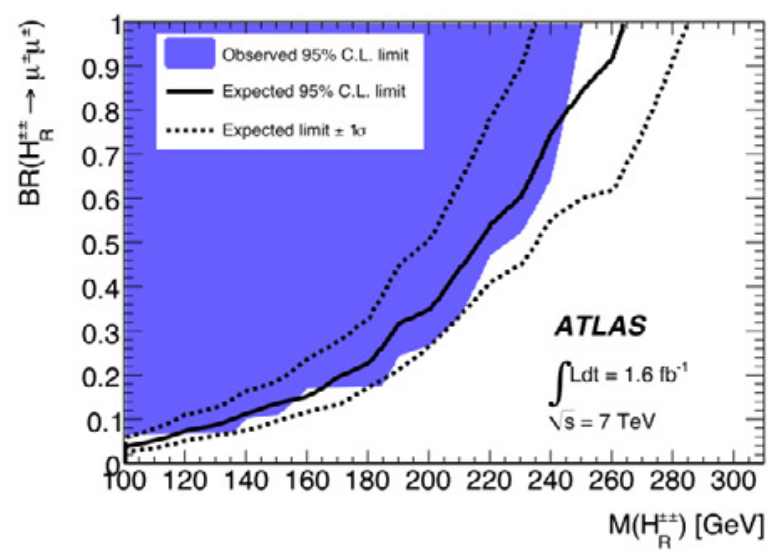

Fig. 22. Exclusion limit at $95 \% \mathrm{CL}$ of the $H^{ \pm \pm}$mass as a function of the branching ratio to muon pairs, $B R\left(H^{ \pm \pm} \rightarrow \mu^{ \pm} \mu^{ \pm}\right)$, for $H_{R}^{ \pm \pm}$bosons. The shaded areas show the observed exclusion region, the solid lines show the expected exclusion region, and the dashed lines show the $\pm 1 \sigma$ variations of the expected exclusion region [22].

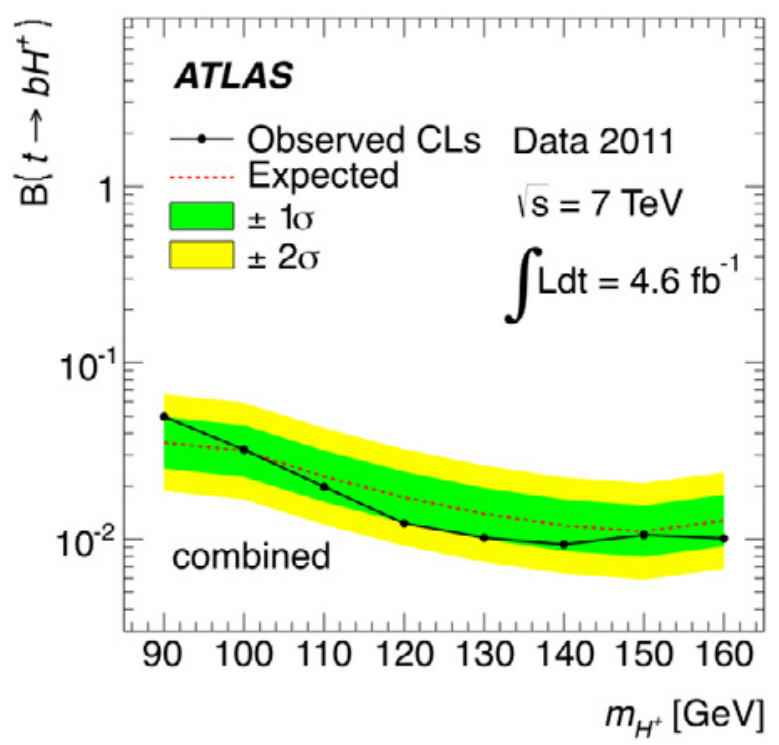

Fig. 23. Observed and expected $95 \% \mathrm{CL}$ exclusion limits on $B\left(t \rightarrow b H^{+}\right)$for charged Higgs boson production from top quark decays as a function of $m_{H^{+}}$, assuming $B(H+\rightarrow \tau v)=100 \%$. Shown are the combination for the lepton+jets, $\tau+$ lepton, and $\tau+$ jets channels [23].

ATLAS has searched for the Standard Model Higgs in the channel $H \rightarrow Z^{-3} \rightarrow \ell^{+} \ell^{-} \ell^{\prime \prime} \ell^{\prime-}$ for $\ell, \ell^{\prime \prime}=e$ or $\mu$ [25]. For 4.8 $\mathrm{fb}^{-1}$ of integrated luminosity, excesses are observed (see Figure 27$)$ at masses $125 \mathrm{GeV}(2.1 \sigma), 244 \mathrm{GeV}(2.2 \sigma)$ and $500 \mathrm{GeV}(2.1 \sigma)$. Once the Look Elsewhere Effect is considered, none of these excesses is significant (Figure 28). 


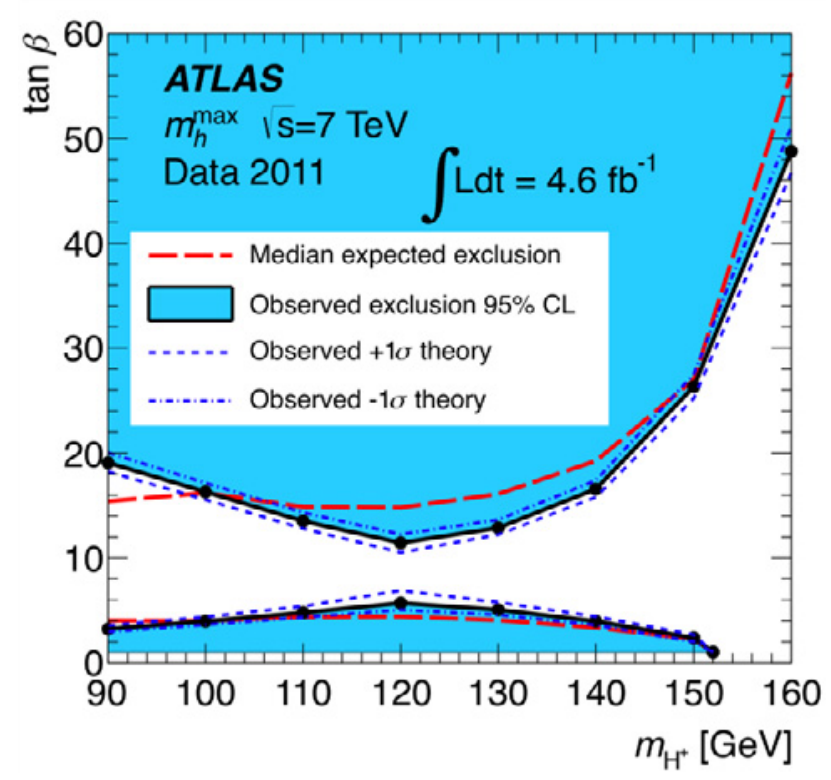

Fig 24. Combined $95 \%$ CL exclusion limits on $\tan \beta$ as a function of $m_{H^{+}}$[23]. Results are shown in the context of the MSSM scenario $m_{\mathrm{h}}{ }^{\mathrm{max}}$ for the region $1<\tan \beta<60$ in which reliable theoretical predictions exist.

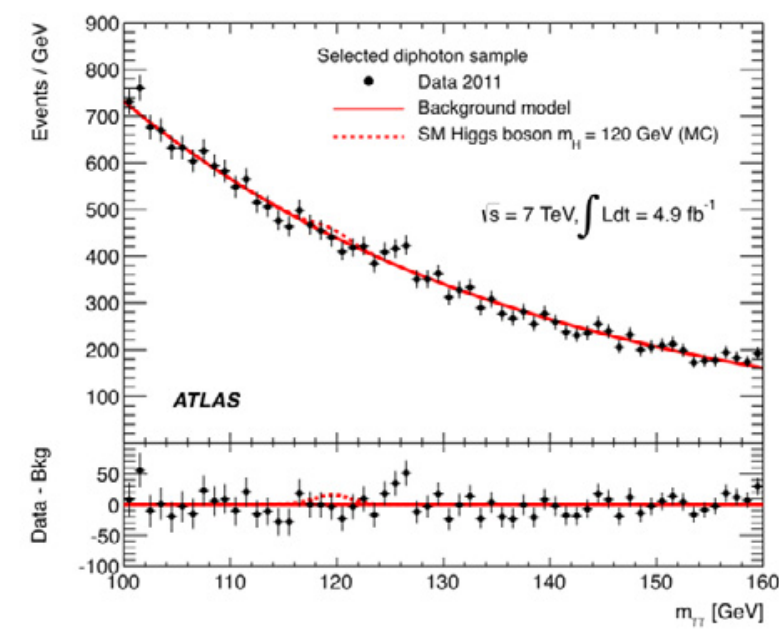

Fig. 25. Invariant mass distribution for the selected data sample overlaid with the total background [24]. The bottom inset displays the residual of the data with respect to the total background. The Higgs boson expectation for a mass hypothesis of $120 \mathrm{GeV}$ corresponding to the Standard Model cross section is also shown.

In the combined search for $H \rightarrow \gamma \gamma, H \rightarrow Z Z^{(*)}, H \rightarrow W W^{(*)}, H \rightarrow \tau^{+} \tau$, and $H \rightarrow b \bar{b}$, the data excess at mass $126 \mathrm{GeV}$ has significance $3.5 \sigma$ [26]. The expected significance in the presence of a Standard Model Higgs with $m_{H}=126 \mathrm{GeV}$ is $2.5 \sigma$. Mass ranges $110.0-117.5,118.5-122.5$, and $129-539 \mathrm{GeV}$ are excluded at $95 \% \mathrm{CL}$. Figures 29 and 30 show the observed and expected upper limits on the production cross section divided by the Standard Model expectation for this combined search, in the full mass range and the low mass range, respectively. 


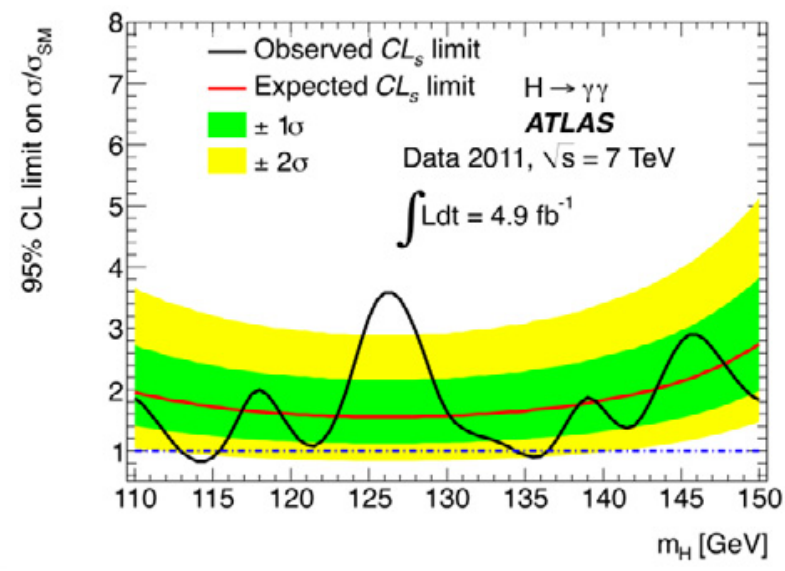

Fig. 26. Observed and expected $95 \%$ CL limits on the Standard Model Higgs boson production cross section normalized to the predicted cross section as a function of $m_{H}$ [24].

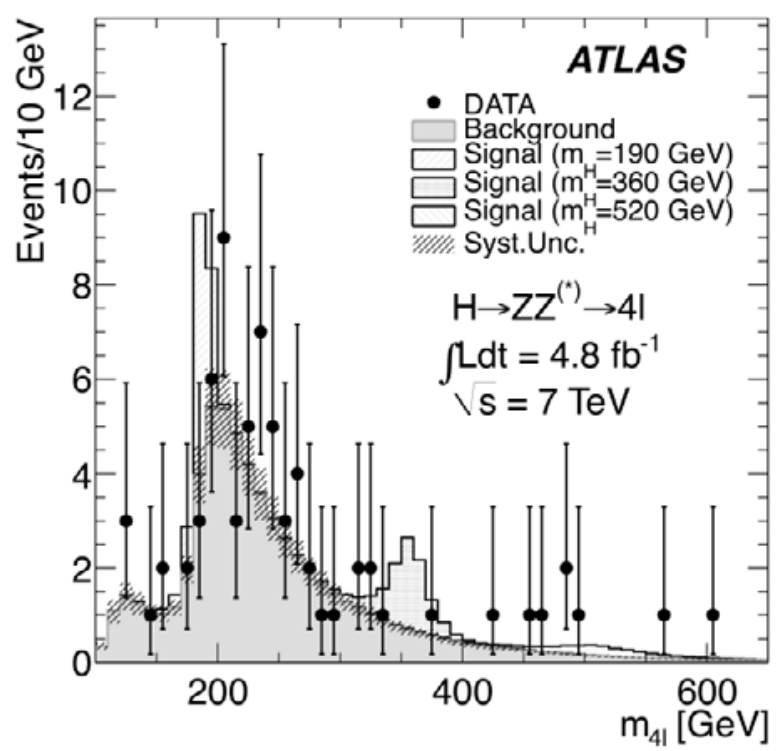

Fig. 27. The $m_{41}$ distribution of candidates compared with the background expectation. Error bars represent $68.3 \%$ central confidence intervals. The signal expectation for several $m_{H}$ hypotheses is also shown [25].

\section{Conclusion}

Results from a representative sample of ATLAS physics analyses are presented. These include searches for new physics, Higgs searches, and Standard Model measurements. 


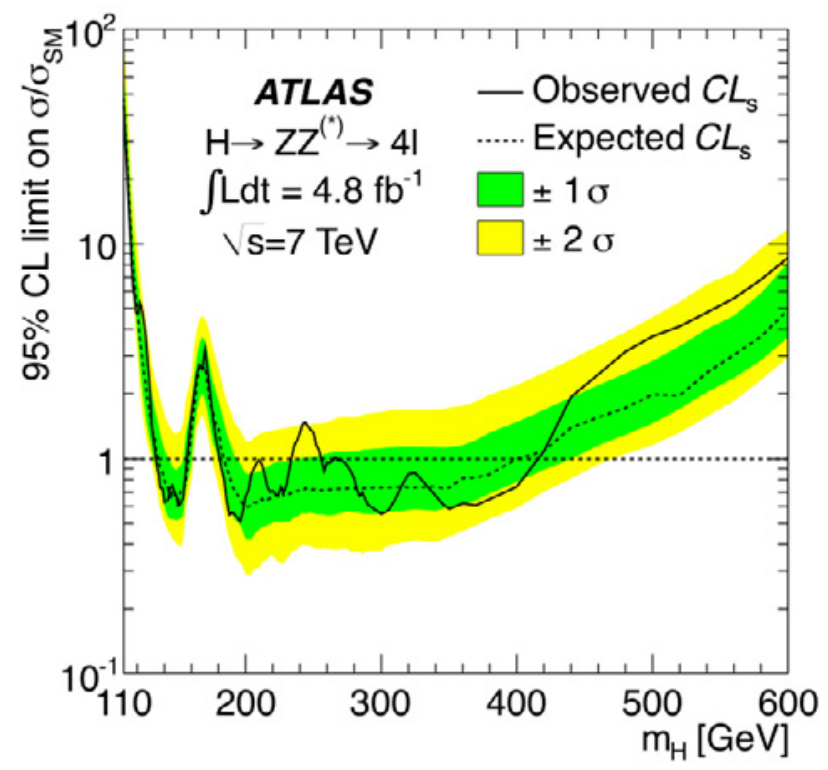

Fig. 28. The observed (full line) and expected (dashed line) 95\% CL upper limits on the Standard Model Higgs boson production cross section as a function of $m_{H}$, divided by the expected Standard Model Higgs boson cross section [25].

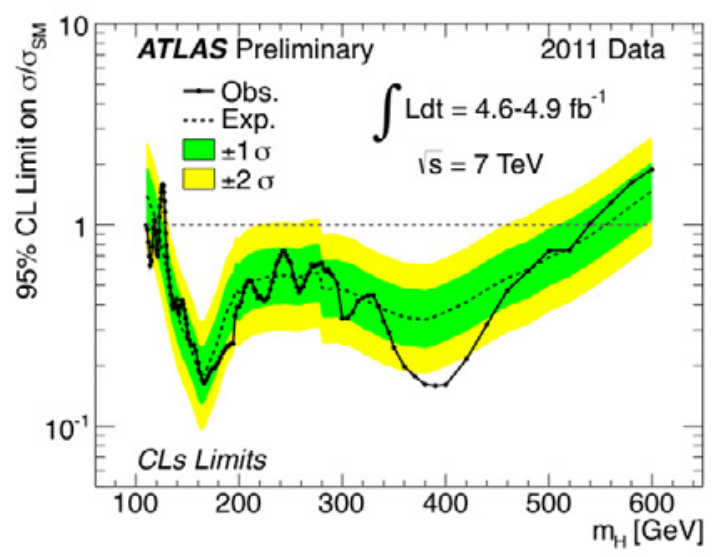

Fig. 29. The observed (full line) and expected (dashed line) 95\% CL combined upper limits on the Standard Model Higgs boson production cross section divided by the Standard Model expectation as a function of $m_{H}$ in the full mass range considered by this analysis. Dotted curves show the median expected limit in the absence of a signal [26].

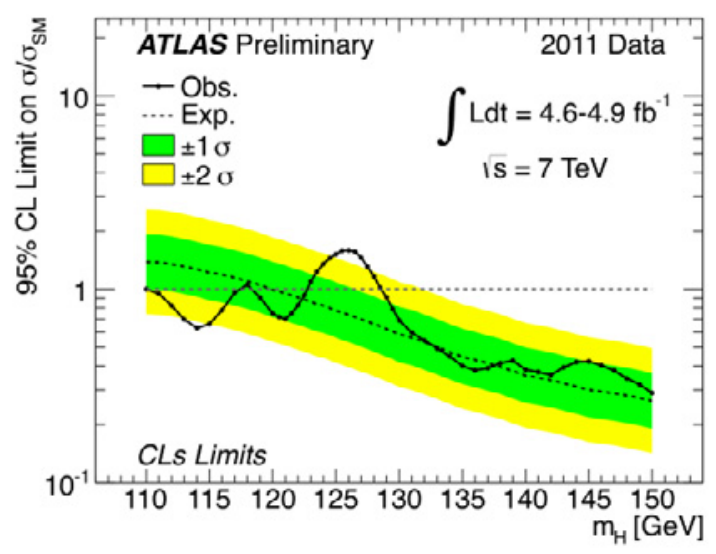

Fig. 30. The observed (full line) and expected (dashed line) $95 \%$ CL combined upper limits on the Standard Model Higgs boson production cross section divided by the Standard Model expectation as a function of $m_{H}$ in the low mass range. Dotted curves show the median expected limit in the absence of a signal [26]. 


\section{References}

1. ATLAS Collaboration, The ATLAS Experiment at the CERN Large Hadron Collider, JINST 3 (2008) S08003.

2. ATLAS Collaboration, Measurement of inclusive two-particle angular correlations with the ATLAS detector at the LHC, JHEP 1205 (2012) 157; arXiv:1203.3549 [hep-ex].

3. ATLAS Collaboration, Measurement of the azimuthal ordering of charged hadrons with the ATLAS detector, CERN-PH-EP2011-197; arXiv:1203.0419 [hep-ex].

4. ATLAS Collaboration, Determination of the strange quark density of the proton from ATLAS measurements of the $W \rightarrow I v$ and $Z \rightarrow$ II cross sections, Phys. Rev. Lett. 109 (2012) 012001; arXiv:1203.4051 [hep-ex].

5. ATLAS Collaboration, Measurement of the production cross section of an isolated photon in association with jets in protonproton collisions at $\sqrt{s}_{\mathrm{s}}=7 \mathrm{TeV}$ with the ATLAS detector, Phys. Rev. D 85 (2012) 092014; arXiv:1203.3161 [hep-ex].

6. ATLAS Collaboration, Measurement of the charge asymmetry in top quark pair production in pp collisions at $\sqrt{\mathrm{s}}_{\mathrm{s}}=7 \mathrm{TeV}$ with the ATLAS detector, Eur. Phys. J. C 72 (2012) 2039; arXiv:1203.4211 [hep-ex].

7. CMS Collaboration, Measurement of the charge asymmetry in top-quark pair production in proton-proton collisions at $\sqrt{s}_{\mathrm{s}}=7$ TeV, Phys. Lett. B 709 (2012) 28; arXiv:1112.5100 [hep-ex].

8. CDF Collaboration, Evidence for a mass dependent forward-backward asymmetry in top quark pair production, Phys. Rev. D 83 (2011) 11203, arXiv:1101.0034 [hep-ex]; D0 Collaboration, Forward-backward asymmetry in top quark-antiquark production, Phys. Rev. D 84 (2011) 112005, arXiv:1107.4995[hep-ex].

9. ATLAS Collaboration, Search for FCNC single top-quark production at $\sqrt{s}_{\mathrm{s}}=7 \mathrm{TeV}$ with the ATLAS detector, Phys. Lett. B 712 (2012) 351-369; arXiv:1203.0529 [hep-ex].

10. ATLAS Collaboration, Search for same-sign top quark production and fourth generation down-type quarks in pp collisions at $\sqrt{\mathrm{s}}_{\mathrm{s}} 7 \mathrm{TeV}$ with the ATLAS detector, JHEP 1204 (2012) 069; arXiv:1202.5520 [hep-ex].

11. ATLAS Collaboration, Search for pair production of a new quark that decays to a $Z$ boson and a bottom quark with the ATLAS detector, Phys. Rev. Lett. 109 (2012) 071801; arXiv:1204.1265 [hep-ex].

12. ATLAS Collaboration, Search for down-type fourth generation quarks with the ATLAS detector in events with one lepton and hadronically decaying W bosons, Phys. Rev. Lett. 109 (2012) 032001; arXiv:1202.6540 [hep-ex].

13. CDF Collaboration, Search for heavy bottomlike quarks decaying to an electron or muon and jets in $p \bar{p}$ collisions at $\sqrt{\mathrm{s}}_{\mathrm{s}}=1.96$ TeV, Phys. Rev. Lett. 106 (2011) 141803.

14. ATLAS Collaboration, Search for pair production of a heavy up-type quark decaying to a $\mathrm{W}$ boson and a $\mathrm{b}$ quark in the lepton+jets channel with the ATLAS detector, Phys. Rev. Lett. 108 (2012) 261802; arXiv:1202.3076 [hep-ex].

15. ATLAS Collaboration, Search for tb resonances in proton-proton collisions at $\sqrt{s}_{\mathrm{s}}=7 \mathrm{TeV}$ with the ATLAS detector, Phys. Rev. Lett. 109 (2012) 081801; arXiv:1205.1016 [hep-ex].

16. ATLAS Collaboration, Search for heavy neutrinos and right-handed $\mathrm{W}$ bosons in events with two leptons and jets in pp collisions at $\sqrt{s}_{\mathrm{s}}=7 \mathrm{TeV}$ with the ATLAS detector, Eur. Phys. J. C 72 (2012) 2056; arXiv:1203.5420 [hep-ex].

17. ATLAS Collaboration, Search for excited leptons in proton-proton collisions at $\sqrt{s}_{\mathrm{s}}=7 \mathrm{TeV}$ with the ATLAS detector, Phys. Rev. D 85 (2012) 072003; arXiv:1201. 3293 [hep-ex].

18. ATLAS Collaboration, Search for second generation scalar leptoquarks in pp collisions at $\sqrt{s}_{\mathrm{s}}=7 \mathrm{TeV}$ with the ATLAS detector, CERN-PH-EP-2012-056; arXiv:1203. 3172 [hep-ex].

19. ATLAS Collaboration, Search for new particles decaying to $\mathrm{ZZ}$ using final states with leptons and jets with the ATLAS detector in $\sqrt{s}_{\mathrm{s}}=7 \mathrm{TeV}$ proton-proton collisions, Phys. Lett. B 712 (2012) 331-350; arXiv:1203.0718 [hep-ex].

20. ATLAS Collaboration, Search for contact interactions in dilepton events from pp collisions at $\sqrt{\mathrm{s}}=$ detector, Phys. Lett. B 712 (2012) 40-58; arXiv:1112.4462 [hep-ex].

21. ATLAS Collaboration, Search for TeV-scale gravity signatures in final states with leptons and jets with the ATLAS detector at $\sqrt{\mathrm{s}}_{\mathrm{s}}=7 \mathrm{TeV}$, CERN-PH-EP-2012-046; arXiv:1204.4646 [hep-ex].

22. ATLAS Collaboration, Search for anomalous production of prompt like-sign muon pairs and constraints on physics beyond the Standard Model with the ATLAS detector, Phys. Rev. D 88 (2012) 032004; arXiv:1201.1091 [hep-ex].

23. ATLAS Collaboration, Search for charged Higgs bosons decaying via $\mathrm{H}^{+} \rightarrow \tau \mathrm{v}$ in top quark pair events using pp collision data at $\sqrt{s}_{\mathrm{s}}=7 \mathrm{TeV}$ with the ATLAS detector, JHEP 1206 (2012) 039; arXiv:1204.2760 [hep-ex].

24. ATLAS Collaboration, Search for the Standard Model Higgs boson in the diphoton decay channel with $4.9 \mathrm{fb}^{-1}$ of pp collisions at $\sqrt{\mathrm{s}}_{\mathrm{s}}=7 \mathrm{TeV}$ with ATLAS, Phys. Rev. Lett. 108 (2012) 111803; arXiv:1202.1414 [hep-ex].

25. ATLAS Collaboration, Search for the Standard Model Higgs boson in the decay channel $H \rightarrow Z Z^{(*)} \rightarrow 4$ I , Phys. Lett. B 710 (2012) 383-402; arXiv:1202.1415 [hep-ex].

26. ATLAS Collaboration, Combined search for the Standard Model Higgs boson using up to $4.9 \mathrm{fb}^{-1}$ of pp collision data at $\sqrt{s}_{\mathrm{s}}=7$ TeV with the ATLAS detector at the LHC, Phys. Lett. B 710 (2012) 49-66; arXiv:1202.1408 [hep-ex]. 